\title{
Manipulation of the Raman process via incoherent pump, tunable intensity, and phase control
}

\author{
Li-Gang Wang, ${ }^{1,2,3}$ Sajid Qamar, ${ }^{1}$ Shi-Yao Zhu, ${ }^{1,2,3}$ and M. Suhail Zubairy ${ }^{1,4,5}$ \\ ${ }^{1}$ Centre for Quantum Physics, COMSATS Institute of Information Technology, Islamabad, Pakistan \\ ${ }^{2}$ Department of Physics, Zhejiang University, Hangzhou 310027, China \\ ${ }^{3}$ Department of Physics, Hong Kong Baptist University, Hong Kong, China \\ ${ }^{4}$ Institute for Quantum Studies and Department of Physics, Texas A\&M University, College Station, Texas 77845, USA \\ ${ }^{5}$ Texas A\&M University at Qatar, P.O. Box 23874, Education City, Doha, Qatar
}

(Received 14 May 2007; published 18 March 2008)

\begin{abstract}
We present a proposal to manipulate the Raman process via incoherent pump, tunable intensity, and phase control of the driving fields. It is found that Raman absorptive peaks can become Raman gain peaks by controlling the incoherent pump, and this property leads to the group velocity of a weak probe pulse from subluminal to superluminal with less absorption or gain. Furthermore, it is also shown that Raman gain peaks can be controlled not only by controlling the intensities of the driving fields but also by adjusting phases of the driving fields, and this leads to the strong negative dispersion with less gain.
\end{abstract}

DOI: 10.1103/PhysRevA.77.033833

PACS number(s): 42.50.Gy, 42.25.Bs

The study of subluminal and superluminal propagation of a light pulse in dispersive media has been extensively investigated in recent years (see some recent reviews [1,2]). One tries to manage the dispersion of the materials in order to control the group velocity of pulse propagation, due to many potential applications in nonlinear optics, high-speed optical switches, optical delay lines, and communication systems [3]. The usual dispersion relation tells us that the strong anomalous (normal) dispersion is always accompanied with a large absorption (gain) peak. Due to the large absorption or gain, the light pulse is strongly distorted within a very short distance. Thus it is interesting, both theoretically and experimentally, to realize subluminal and superluminal light propagation with negligible absorption or gain. Harris et al. [4] predicted the possibility of ultraslow propagation via electromagnetically induced transparency (EIT), which was realized experimentally in an ultracold atomic gas by Hau et al. [5]. Many other experiments on subluminal propagation were reported by using an atomic gas [6-9] and solid-state materials $[10,11]$. In 2000, based on the earlier suggestion of Steinberg and Chiao [12], Wang et al. [13] used two pump fields to realize a double-peaked Raman gain profile, which leads to superluminal propagation with less gain.

In recent years, a number of methods and proposals are presented for achieving the switching from subluminal to superluminal propagations $[3,14-22]$ in a single system. Talukder et al. [14] realized femtosecond laser pulses propagating from superluminal to subluminal group velocities in a resonantly absorbing dye by changing dye concentrations. Shimizu et al. [15] also observed the transition from superluminal to subluminal propagation in a high-finesse microcavity containing a few cold atoms. Kim et al. [3] reported on a simple two-level atomic system, which can lead to a switching from fast to slow pulse propagation based on electromagnetically induced absorption and EIT by changing the intensity of the coherent driving field. Agarwal et al. [16] proposed a scheme for obtaining the light propagation from superluminal to subluminal velocity by changing the intensity of the coupling field between two lower levels in a $\Lambda$-type three-level atomic system. Agarwal et al. [17] further presented a scheme for manipulating the Raman process by using a coherent controlling field that leads to the splitting of the Raman gain peak into a doublet and anomalous dispersion in the region between the two gain peaks. The group velocity of a weak pulse can also be manipulated by controlling the phases of two weak optical fields applied to a $V$-shaped three-level system proposed by Bortman-Arbiv et al. [20]. Sahrai et al. [21] suggested tunable control of the group velocity from subluminal to superluminal by changing the phase of one of the deriving fields in a four-level atomic system. More recently, an incoherent pump field is used to control the light propagation from subluminal to superluminal [22].

As we know, the Raman gain scheme has several advantages such as excellent signal-to-noise ratio and widely tunability compared with the conventional EIT scheme [23]. One of our motivations in this paper is whether we can realize the switching of the subluminal to superluminal light propagation by manipulating the Raman process using different controlling parameters, such as the incoherent pumping and tunable intensity control, only within one atomic system. Another important property is that the Raman process could be changed by phase control. Our scheme is different from the proposal of the $N$ configuration [17] where the splitting of two Raman peaks is only controlled by the intensity of the coherent controlling field without phase dependence, whereas in our scheme the Raman peaks are sensitively dependent on the quantum interference between different twophoton pathways. In this paper, we show that the Raman process can be controlled by incoherent pump, which leads to the transition from the subluminal to superluminal propagation for the probe pulse with less absorption or gain. We also show that the Raman process can be manipulated by the intensities or the phases of the controlling fields that leads to the splitting of Raman gain peak or the disappearance of the two Raman gain peaks. Compared with the previous proposals $[16,20,21]$, the advantage of our scheme is that we do not use the controlling parameter applied on the dipole forbidden transition.

We consider an atomic system as shown in Fig. 1(a). The atom has three nondegenerate ground levels $|d\rangle,|b\rangle$, and $|e\rangle$ and two excited states $|a\rangle$ and $|c\rangle$. The dipole-allowed tran- 


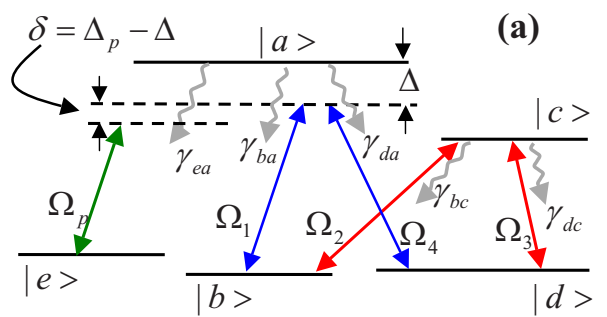

(b)

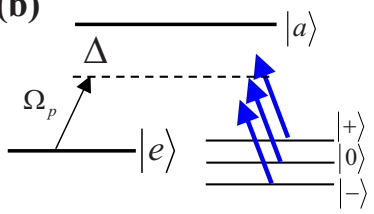

FIG. 1. (Color online) (a) A five-level atomic system. Two coherently driving fields as the controlling fields with Rabi frequencies $\Omega_{2}$ and $\Omega_{3}$ are indicated by the arrows; the other two pumping fields with Rabi frequencies $\Omega_{1}$ and $\Omega_{4}$ with the detuning $\Delta$ are indicated by the arrows; notice that the incoherent pumping is not shown. (b) The schematic of the equivalent energy levels in the dressed-state picture, and arrows denote the three Raman channels.

sitions $|b\rangle-|a\rangle,|b\rangle-|c\rangle,|d\rangle-|c\rangle$, and $|d\rangle-|a\rangle$ are driven by four coherent fields with Rabi frequencies $\Omega_{1}, \Omega_{2}, \Omega_{3}$, and $\Omega_{4}$, respectively, where the deriving fields associated with $\Omega_{2}$ and $\Omega_{3}$ acting as the controlling fields are assumed to be resonant with the transitions $|b\rangle-|c\rangle$ and $|d\rangle-|c\rangle$. The probe field $E_{p}$ is coupled to the dipole-allowed transition $|e\rangle-|a\rangle$ with the corresponding Rabi frequency $\Omega_{p}=E_{p} u_{a e} / \hbar$, where $u_{a e}$ is the induced atomic dipole moment. At the same time the transition $|e\rangle-|a\rangle$ is incoherently pumped with a rate $\Lambda$ by incoherent pumping. The atom decays from the exited state $|a\rangle$ to the lower-lying level $|b\rangle(|d\rangle$ and $|e\rangle)$ at the spontaneous emission rate $\gamma_{b a}\left(\gamma_{d a}\right.$ and $\left.\gamma_{e a}\right)$ and from the exited state $|c\rangle$ to the lower levels $|b\rangle(|d\rangle)$ at the spontaneous emission rate $\gamma_{b c}\left(\gamma_{d c}\right)$. It should be mentioned that our proposal may be realized in the closed $M$-type five-level atomic configuration by using the cesium (Cs) atom vapor, similar to Ref. [24], in which Li et al. used an $M$-type five-level Cs atom to achieve a large index of refraction with vanishing absorption. Here in our proposal, the levels $|b\rangle,|d\rangle$, and $|c\rangle$ can correspond to $\left|6 S_{1 / 2}, F=3, M_{F}=+1\right\rangle, \mid 6 S_{1 / 2}, F=3, M_{F}$ $=+3\rangle$, and $\left|6 P_{3 / 2}, F=2, M_{F}=+2\right\rangle$, respectively; and the levels $|a\rangle$ and $|e\rangle$ can correspond to $\left|6 P_{3 / 2}, F=4\right\rangle$ and $\mid 6 S_{1 / 2}, F$ $=4\rangle$, respectively.

The Hamiltonian for this system in the dipole and the rotating wave approximation can be written as

$$
H=H_{0}+H_{I},
$$

where $H_{0}=\Sigma_{i} \hbar \omega_{i}|i\rangle\langle i|(i=a, b, c, d, e)$ is the self-energy, and $\omega_{i}$ corresponds to the energy of state $|i\rangle$; and the interaction Hamiltonian is given by

$$
\begin{aligned}
H_{I}= & -\frac{\hbar}{2}\left[\Omega_{1} e^{-i \nu_{1} t}|a\rangle\left\langle b\left|+\Omega_{2} e^{-i \nu_{2} t}\right| c\right\rangle\left\langle b\left|+\Omega_{3} e^{-i \nu_{3} t}\right| c\right\rangle\langle d|\right. \\
& \left.+\Omega_{4} e^{-i \nu_{4} t}|a\rangle\left\langle d\left|+\Omega_{p} e^{-i \nu_{p} t}\right| a\right\rangle\langle e|+\text { H.c. }\right],
\end{aligned}
$$

where $\nu_{i}(i=1,2,3,4)$ and $\nu_{p}$ are the angular frequencies of the driving and probe fields, respectively.
Starting from the general density-matrix equations of motion, and transforming to appropriate rotating frames by using the relations

$$
\begin{gathered}
\rho_{a b}=e^{-i \nu_{1} t} \widetilde{\rho}_{a b}, \quad \rho_{a c}=e^{-i \nu_{1} t+i \nu_{2} t} \widetilde{\rho}_{a c}, \quad \rho_{a d}=e^{-i \nu_{4} t} \widetilde{\rho}_{a d}, \\
\rho_{a e}=e^{-i \omega_{a e} t} \widetilde{\rho}_{a e}, \\
\rho_{b c}=e^{-i \nu_{2} t} \widetilde{\rho}_{b c}, \quad \rho_{b d}=e^{i \nu_{2} t-i \nu_{3} t} \widetilde{\rho}_{b d}, \quad \rho_{b e}=e^{i \nu_{1} t-i \omega_{a e} t} \widetilde{\rho}_{b e}, \\
\rho_{c d}=e^{-i \nu_{3} t} \widetilde{\rho}_{c d}, \\
\rho_{c e}=e^{-i \nu_{3} t+i \nu_{4} t-i \omega_{a e} t} \widetilde{\rho}_{c e}, \quad \rho_{d e}=e^{i \nu_{4} t-i \omega_{a e} t} \widetilde{\rho}_{d e},
\end{gathered}
$$

and eliminating $\widetilde{\rho}_{a a}$ through the population conservation rule for the closed atomic system $\widetilde{\rho}_{a a}+\widetilde{\rho}_{b b}+\widetilde{\rho}_{c c}+\widetilde{\rho}_{d d}+\widetilde{\rho}_{e e}=1$, we obtain

$$
\begin{aligned}
& \dot{\tilde{\rho}}_{a b}+\frac{i}{2} \Omega_{1}=-\left(\Gamma_{a b}+\frac{1}{2} \Lambda+i \Delta_{1}\right) \widetilde{\rho}_{a b}+\frac{i}{2} \Omega_{1}\left(2 \widetilde{\rho}_{b b}+\widetilde{\rho}_{c c}+\widetilde{\rho}_{d d}\right. \\
& \left.+\widetilde{\rho}_{e e}\right)-\frac{i}{2} \Omega_{2} \widetilde{\rho}_{a c}+\frac{i}{2} \Omega_{4} \widetilde{\rho}_{d b}+\frac{i}{2} \Omega_{p} e^{-i \Delta_{p} t} \widetilde{\rho}_{e b} \\
& \dot{\tilde{\rho}}_{a c}=-\left[\Gamma_{a c}+\frac{1}{2} \Lambda+i\left(\Delta_{1}-\Delta_{2}\right)\right] \widetilde{\rho}_{a c}+\frac{i}{2} \Omega_{1} \widetilde{\rho}_{b c}-\frac{i}{2} \Omega_{2}^{*} \widetilde{\rho}_{a b} \\
& -\frac{i}{2} \Omega_{3}^{*} \widetilde{\rho}_{a d}+\frac{i}{2} \Omega_{4} \widetilde{\rho}_{d c}+\frac{i}{2} \Omega_{p} e^{-i \Delta_{p} t} \widetilde{\rho}_{e c}, \\
& \dot{\tilde{\rho}}_{a d}+\frac{i}{2} \Omega_{4}=-\left(\Gamma_{a d}+\frac{1}{2} \Lambda+i \Delta_{4}\right) \widetilde{\rho}_{a d}+\frac{i}{2} \Omega_{4}\left(\widetilde{\rho}_{b b}+\widetilde{\rho}_{c c}+2 \widetilde{\rho}_{d d}\right. \\
& \left.+\widetilde{\rho}_{e e}\right)+\frac{i}{2} \Omega_{1} \tilde{\rho}_{b d}-\frac{i}{2} \Omega_{3} \widetilde{\rho}_{a c}+\frac{i}{2} \Omega_{p} e^{-i \Delta_{p} t} \widetilde{\rho}_{e d} \\
& \dot{\tilde{\rho}}_{a e}+\frac{i}{2} \Omega_{p} e^{-i \Delta_{p} t}=-\left(\Gamma_{a e}+\Lambda\right) \tilde{\rho}_{a e}+\frac{i}{2} \Omega_{p} e^{-i \Delta_{p} t}\left(\widetilde{\rho}_{b b}+\widetilde{\rho}_{c c}+\widetilde{\rho}_{d d}\right. \\
& \left.+2 \widetilde{\rho}_{e e}\right)+\frac{i}{2} \Omega_{1} \tilde{\rho}_{b e}+\frac{i}{2} \Omega_{4} \tilde{\rho}_{d e}, \\
& \dot{\tilde{\rho}}_{b b}-\gamma_{b a}=-\gamma_{b a} \widetilde{\rho}_{b b}+\left(\gamma_{b c}-\gamma_{b a}\right) \tilde{\rho}_{c c}-\gamma_{b a} \tilde{\rho}_{d d}-\gamma_{b a} \tilde{\rho}_{e e} \\
& +\frac{i}{2} \Omega_{1}^{*} \tilde{\rho}_{a b}-\frac{i}{2} \Omega_{1} \tilde{\rho}_{b a}+\frac{i}{2} \Omega_{2}^{*} \tilde{\rho}_{c b}-\frac{i}{2} \Omega_{2} \tilde{\rho}_{b c}, \\
& \dot{\tilde{\rho}}_{b c}=-\left(\Gamma_{b c}-i \Delta_{2}\right) \widetilde{\rho}_{b c}+\frac{i}{2} \Omega_{1}^{*} \widetilde{\rho}_{a c}+\frac{i}{2} \Omega_{2}^{*}\left(\widetilde{\rho}_{c c}-\widetilde{\rho}_{b b}\right)-\frac{i}{2} \Omega_{3}^{*} \widetilde{\rho}_{b d},
\end{aligned}
$$




$$
\begin{gathered}
\dot{\tilde{\rho}}_{b d}=-\left[\Gamma_{b d}+i\left(\Delta_{3}-\Delta_{2}\right)\right] \widetilde{\rho}_{b d}+\frac{i}{2} \Omega_{1}^{*} \tilde{\rho}_{a d}+\frac{i}{2} \Omega_{2}^{*} \widetilde{\rho}_{c d}-\frac{i}{2} \Omega_{3} \widetilde{\rho}_{b c} \\
-\frac{i}{2} \Omega_{4} \widetilde{\rho}_{b a}, \\
\dot{\tilde{\rho}}_{b e}=-\left(\Gamma_{a e}+\frac{1}{2} \Lambda-i \Delta_{1}\right) \widetilde{\rho}_{b e}+\frac{i}{2} \Omega_{1}^{*} \widetilde{\rho}_{a e}+\frac{i}{2} \Omega_{2}^{*} \widetilde{\rho}_{c e} \\
\quad-\frac{i}{2} \Omega_{p} e^{-i \Delta_{p} t} \widetilde{\rho}_{b a}, \\
\dot{\tilde{\rho}}_{c c}=-\left(\gamma_{b c}+\gamma_{d c}\right) \widetilde{\rho}_{c c}+\frac{i}{2} \Omega_{2} \widetilde{\rho}_{b c}-\frac{i}{2} \Omega_{2}^{*} \widetilde{\rho}_{c b}+\frac{i}{2} \Omega_{3} \widetilde{\rho}_{d c} \\
-\frac{i}{2} \Omega_{3}^{*} \widetilde{\rho}_{c d},
\end{gathered}
$$$$
\dot{\tilde{\rho}}_{c d}=-\left(\Gamma_{c d}+i \Delta_{3}\right) \widetilde{\rho}_{c d}+\frac{i}{2} \Omega_{3}\left(\widetilde{\rho}_{d d}-\widetilde{\rho}_{c c}\right)+\frac{i}{2} \Omega_{2} \widetilde{\rho}_{b d}-\frac{i}{2} \Omega_{4} \widetilde{\rho}_{c a},
$$

$$
\begin{aligned}
\dot{\tilde{\rho}}_{c e}= & -\left[\Gamma_{c e}+\frac{1}{2} \Lambda+i\left(\Delta_{3}-\Delta_{4}\right)\right] \widetilde{\rho}_{c e}+\frac{i}{2} \Omega_{2} \widetilde{\rho}_{b e}+\frac{i}{2} \Omega_{3} \widetilde{\rho}_{d e} \\
& -\frac{i}{2} \Omega_{p} e^{-i \Delta_{p} t} \widetilde{\rho}_{c a}
\end{aligned}
$$$$
\dot{\tilde{\rho}}_{d d}-\gamma_{d a}=-\gamma_{d a} \widetilde{\rho}_{b b}+\left(\gamma_{d c}-\gamma_{d a}\right) \widetilde{\rho}_{c c}-\gamma_{d a} \widetilde{\rho}_{d d}-\gamma_{d a} \widetilde{\rho}_{e e}
$$$$
-\frac{i}{2} \Omega_{3} \tilde{\rho}_{d c}+\frac{i}{2} \Omega_{3}^{*} \tilde{\rho}_{c d}-\frac{i}{2} \Omega_{4} \tilde{\rho}_{d a}+\frac{i}{2} \Omega_{4}^{*} \tilde{\rho}_{a d}
$$$$
\dot{\tilde{\rho}}_{d e}=-\left(\Gamma_{d e}+\frac{1}{2} \Lambda-i \Delta_{4}\right) \tilde{\rho}_{d e}+\frac{i}{2} \Omega_{3}^{*} \tilde{\rho}_{c e}+\frac{i}{2} \Omega_{4}^{*} \widetilde{\rho}_{a e}
$$$$
-\frac{i}{2} \Omega_{p} e^{-i \Delta_{p} t} \widetilde{\rho}_{d a}
$$$$
\dot{\tilde{\rho}}_{e e}-\left(\gamma_{e a}+\Lambda\right)=-\left(\gamma_{e a}+\Lambda\right)\left(\widetilde{\rho}_{b b}+\tilde{\rho}_{c c}+\tilde{\rho}_{d d}+\tilde{\rho}_{e e}\right)
$$$$
+\frac{i}{2} \Omega_{p}^{*} e^{i \Delta_{p} t} \widetilde{\rho}_{a e}-\frac{i}{2} \Omega_{p} e^{-i \Delta_{p} t} \widetilde{\rho}_{e a} .
$$

Here $\Delta_{1}=\omega_{a b}-\nu_{1}, \Delta_{2}=\omega_{c b}-\nu_{2}, \Delta_{3}=\omega_{c d}-\nu_{3}, \Delta_{4}=\omega_{a d}-\nu_{4}$, $\Delta_{p}=\nu_{p}-\omega_{a e}$, and $\Gamma_{\alpha \beta}=\Sigma_{i}\left(\gamma_{i \alpha}+\gamma_{i \beta}\right)+\gamma_{\text {coll }}$, where $\gamma_{\alpha \beta}$ is the decay rate from the level $|\beta\rangle$ to $|\alpha\rangle$ and $\gamma_{\text {coll }}$ is the collisional decay rate. In the above equations, we have used the required condition $\nu_{1}+\nu_{3}=\nu_{2}+\nu_{4}$ for the driving fields, which is equivalent to $\Delta_{1}+\Delta_{3}=\Delta_{2}+\Delta_{4}$. As the four driving fields form a closed-loop coherent pumping, the phases of $\Omega_{1}, \Omega_{2}, \Omega_{3}$, and $\Omega_{4}$ can be imparted to any one of them and that will not change the result of the calculation. Therefore, for the rest of discussion, we assume the Rabi frequencies $\Omega_{1}, \Omega_{3}$, and $\Omega_{4}$ to be real and assume $\Omega_{2}$ to carry the phase $\phi_{0}$, i.e., $\Omega_{2}$ $=\left|\Omega_{2}\right| e^{-i \phi_{0}}$. Furthermore, Eqs. (4) for the matrix elements can be written in a compact form

$$
\dot{R}+\Sigma=M R,
$$

where

$$
\begin{aligned}
R= & \left(\widetilde{\rho}_{a b}, \widetilde{\rho}_{a c}, \widetilde{\rho}_{a d}, \widetilde{\rho}_{a e}, \widetilde{\rho}_{b a}, \widetilde{\rho}_{b b}, \widetilde{\rho}_{b c}, \widetilde{\rho}_{b d}, \widetilde{\rho}_{b e}, \widetilde{\rho}_{c a}, \widetilde{\rho}_{c b}, \widetilde{\rho}_{c c}, \widetilde{\rho}_{c d}, \widetilde{\rho}_{c e},\right. \\
& \left.\tilde{\rho}_{d a}, \widetilde{\rho}_{d b}, \widetilde{\rho}_{d c}, \widetilde{\rho}_{d d}, \widetilde{\rho}_{d e}, \widetilde{\rho}_{e a}, \widetilde{\rho}_{e b}, \widetilde{\rho}_{e c}, \widetilde{\rho}_{e d}, \widetilde{\rho}_{e e}\right)^{T}
\end{aligned}
$$

is a vector containing the density matrix elements and

$$
\begin{aligned}
\Sigma= & \left(\frac{i}{2} \Omega_{1}, 0, \frac{i}{2} \Omega_{4}, \frac{i}{2} \Omega_{p} e^{-i \Delta_{p} t},-\frac{i}{2} \Omega_{1}^{*},-\gamma_{b a}, 0,0,0,0,0,0,0,0,\right. \\
& \left.-\frac{i}{2} \Omega_{4}^{*}, 0,0,-\gamma_{d a}, 0,-\frac{i}{2} \Omega_{p}^{*} e^{i \Delta_{p} t}, 0,0,0,-\gamma_{e a}-\Lambda\right)^{T}
\end{aligned}
$$

is a constant vector. Here the symbol " $T$ " denotes the matrix transpose. The matrix $M$ can be obtained from the coefficients of Eqs. (4). Both the vector $\Sigma$ and matrix $M$ can be separated into terms with different time dependences [25-27], i.e.,

$$
\begin{gathered}
\Sigma=\Sigma_{0}+\Omega_{p} e^{-i \Delta_{p} t} \Sigma_{+1}+\Omega_{p}^{*} e^{i \Delta_{p} t \Sigma_{-1},} \\
M=M_{0}+\Omega_{p} e^{-i \Delta_{p} t} M_{+1}+\Omega_{p}^{*} e^{i \Delta_{p} t} M_{-1},
\end{gathered}
$$

where $\Sigma_{0}, \Sigma_{ \pm 1}, M_{0}, M_{ \pm 1}$ are all constant matrices. Using these definitions, we obtain

$$
\begin{aligned}
\dot{R} & +\Sigma_{0}+\Omega_{p} e^{-i \Delta_{p} t \Sigma_{+1}}+\Omega_{p}^{*} e^{i \Delta_{p} t \Sigma_{-1}} \\
& =\left(M_{0}+\Omega_{p} e^{-i \Delta_{p} t} M_{+1}+\Omega_{p}^{*} e^{i \Delta_{p} t} M_{-1}\right) R .
\end{aligned}
$$

Applying the Floquet theorem it is easy to find that the stationary solution $R$ will have only terms at the harmonics of the detuning $\Delta_{p}$. We focus on the case where the probe field $E_{p}$ is so weak that the Floquet harmonic expansion can be truncated at the first order,

$$
R=R_{0}+\Omega_{p} e^{-i \Delta_{p} t} R_{+1}+\Omega_{p}^{*} e^{i \Delta_{p} t} R_{-1} .
$$

On substituting Eq. (10) into Eq. (9) and equating the coefficients of the different harmonics of $\Delta_{p}$ and corresponding powers of $\Omega_{p}$, we obtain the solution for $R_{0}$ and $R_{+1}$ as

$$
\begin{gathered}
R_{0}=M_{0}^{-1} \Sigma_{0}, \\
R_{+1}=\left(M_{0}+i \Delta_{p}\right)^{-1}\left(\Sigma_{+1}-M_{+1} R_{0}\right) .
\end{gathered}
$$

The linear susceptibility $\chi$ of the weak probe field in the present system is given by [28]

$$
\begin{gathered}
\chi=\frac{2 N u_{e a} \rho_{a e}}{\varepsilon_{0} E_{p}} e^{i \nu_{p} t}=\frac{2 N u_{e a} \tilde{\rho}_{a e}}{\varepsilon_{0} E_{p}} e^{i \Delta_{p} t} \\
\propto \frac{2 N u_{e a}}{\varepsilon_{0} E_{p}} \Omega_{p}\left[R_{+1}\right]_{4}=\frac{2 N\left|u_{e a}\right|^{2}}{\varepsilon_{0} \hbar}\left[R_{+1}\right]_{4},
\end{gathered}
$$

where $\left[R_{+1}\right]_{4}$ denotes the fourth component of $R_{+1}$ and $N$ is the atomic density. From Eq. (13), we can easily obtain the index of refraction and absorption from the real and imaginary part of $\chi$, respectively. Moreover, if all the fields have phase dependence, only the collective phase would be important and this collective phase can be easily determined to be 
$\phi_{0}=\left(\phi_{1}+\phi_{3}\right)-\left(\phi_{2}+\phi_{4}\right)$ by repeating the susceptibility calculation and noting that the Rabi frequencies are complex in general, where $\phi_{i}$ is the phase of the complex Rabi frequency $\Omega_{i}(i=1,2,3,4)$. In principle, we could analytically solve Eqs. (11) and (12) to obtain the expression for the linear susceptibility $\chi$. However, in the current problem it is difficult to obtain the inverse matrices of the $24 \times 24$ matrices. Therefore, we use the numerical simulation to investigate the properties of the linear susceptibility in our five-level atomic scheme.

It is well know that the propagation of the probe pulse can be described by its group velocity. For the narrow-spectral probe pulse, the group velocity can be given by [17]

$$
v_{g}=\frac{c}{1+2 \pi \operatorname{Re}\left[\chi\left(\nu_{p}\right)\right]+2 \pi \nu_{p} \partial \operatorname{Re}\left[\chi\left(\nu_{p}\right)\right] / \partial \nu_{p}},
$$

where $c$ is the speed of light in vacuum, and the refractive index is $n_{r}=1+2 \pi \operatorname{Re}\left[\chi\left(\nu_{p}\right)\right]$. For normal dispersion $\left(\partial \operatorname{Re}\left[\chi\left(\nu_{p}\right)\right] / \partial \nu_{p}>0\right)$, the group velocity is smaller than the phase velocity $\left(v_{g}<c / n_{r}\right)$, and even extremely slow [4-11]; while for anomalous dispersion $\left(\partial \operatorname{Re}\left[\chi\left(\nu_{p}\right)\right] / \partial \nu_{p}<0\right)$, the group velocity can be larger than the phase velocity $\left(v_{g}\right.$ $>c / n_{r}$ ), the speed of light $c$ in vacuum or even becomes negative $\left(v_{g}<0\right)[12,13,19]$. Obviously, when the weak probe pulse passes through an atomic medium with the length $L$, it suffers a time delay $\tau=\tau_{0}\left(n_{g}-1\right)$ compared with the case when it passes though the same distance in vacuum (here $\tau_{0}=L / c$ is the time delay in vacuum). For the sake of simplicity in the following discussion, we define a quantity $\eta=n_{g}-1=2 \pi \operatorname{Re}\left[\chi\left(\nu_{p}\right)\right]+2 \pi \nu_{p} \partial \operatorname{Re}\left[\chi\left(\nu_{p}\right)\right] / \partial \nu_{p}$ such that the condition for subluminal light propagation is $\eta>0$ and the condition for superluminal propagation is $\eta<0$. Here $n_{g}$ is the group index.

In what follows, we present our numerical results. Without loss of generality, we take all the spontaneous-emission rates $\gamma_{b a}=\gamma_{d a}=\gamma_{e a}=\gamma_{b c}=\gamma_{d c}=\gamma$, and assume the detunings of $\Omega_{1}$ and $\Omega_{4}$ to be $\Delta_{1}=\Delta_{4}=\Delta \neq 0$ and the detunings of the controlling fields $\Omega_{2}$ and $\Omega_{3}$ to be $\Delta_{2}=\Delta_{3}=0$. We also take the collisional decay rate $\gamma_{\text {coll }}=0.01 \gamma$. We assume the center angular frequency $\nu_{p}$ of the probe pulse to be $\nu_{p}=10^{8} \gamma$, which is the case for the cesium atomic system $[13,24]$ and the ${ }^{87} \mathrm{Rb}$ atomic system [29]. In all the following figures, $\chi$ and $\eta$ are plotted in the units of $2 N\left|u_{e a}\right|^{2} /\left(\varepsilon_{0} \hbar\right)$, and the variable $\delta \equiv \Delta_{p}-\Delta$ is the two-photon detuning.

First, let us consider the effect of the incoherent pumping $\Lambda$ on the susceptibility $\chi$ and the value of $\eta$. Figures 2(a)-2(c) show the typical effect of different incoherent pumping $\Lambda$ on $\chi$ and $\eta$ under the parameters $\Omega_{1}=\Omega_{4}=2 \gamma$, $\left|\Omega_{2}\right|=\Omega_{3}=5 \gamma, \Delta=10 \gamma$, and $\phi_{0}=0$. It is clearly seen that when $\Lambda=0$ (i.e., without the incoherent pump), there are two Raman absorptive peaks [indicated by the two arrows in Fig. 2(a)], which leads to a subluminal propagation of the probe pulse with less absorption (below we will discuss how the splitting of these two Raman peaks can be controlled by the intensity and phase of the controlling fields $\Omega_{2}$ and $\Omega_{3}$ ). As the value of $\Lambda$ increases, two Raman absorptive peaks gradually become two Raman gain peaks due to the atomic population transfer [see Figs. 2(a)-2(c)]. Therefore, the change of
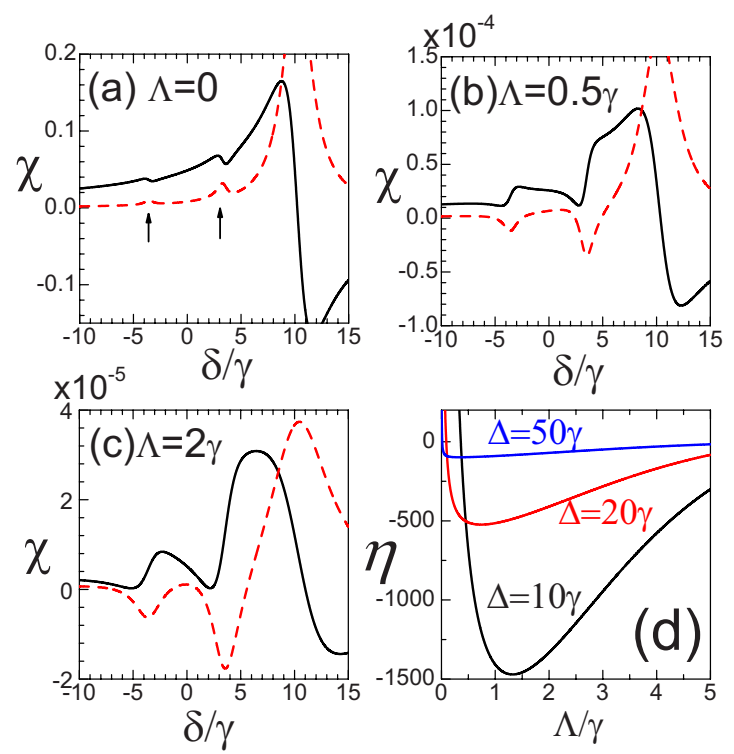

FIG. 2. (Color online) Effect of the incoherent pumping rate $\Lambda$ on the susceptibility $\chi$ and the value of $\eta$ under the parameters $\Omega_{1}=\Omega_{4}=2 \gamma,\left|\Omega_{2}\right|=\Omega_{3}=5 \gamma, \Delta=10 \gamma$, and $\phi_{0}=0$. In (a) $-(\mathrm{c})$ the solid curve denotes the real part of $\chi$, and the dashed curve denotes the imaginary part of $\chi$; (d) the dependence of the value of $\eta$ (at the frequency of the two-photon resonance) on the incoherent pump rate under different detunings: $\Delta=10 \gamma, \Delta=20 \gamma$, and $\Delta=50 \gamma$.

the refractive index near the frequency of the two-photon resonance $\delta=0$ leads to the negative dispersion with much less gain or absorption. This indicates that the propagation of the probe pulse changes from subluminal to superluminal with less absorption or gain. From Fig. 2(d), it is seen that the switching from subluminal $(\eta>0)$ to superluminal $(\eta$ $<0)$ propagation occurs for the appropriate value of the incoherent pump rate $\Lambda$; and the value of $\eta$ (related with the group index) becomes very negative under the suitable value of $\Lambda$ for different detuning $\Delta$. From Fig. 2(d), it is clear that for the large detuning $\Delta$, the anomalous dispersion can be obtained even for the much smaller value of the incoherent pumping rate $(\Lambda \ll \gamma)$. It should be mentioned that there is an absorptive peak near $\delta=10 \gamma$ in Figs. 2(a) $-2(\mathrm{c})$, due to the one-photon resonance.

We know from earlier research works $[30,31]$ that one has used the incoherent pumping field to establish the atomic coherence or interference of pathways. However, in our case the incoherent pumping field leads to the atomic population transfer from the ground state $|e\rangle$ back to the level $|a\rangle$, and then to the linkage $|b\rangle \leftrightarrow|c\rangle \leftrightarrow|d\rangle$ via corresponding spontaneous-decay channels as well. In order to further understand the physical role of the incoherent pumping, in Fig. 3 , we depict the dependence of the probe gain $\operatorname{Im}\left[\rho_{a e}\right]$ on the incoherent pumping at the frequency of the two-photon resonance $\delta=0$ for different detuning: $\Delta=10 \gamma, \Delta=20 \gamma$, and $\Delta$ $=50 \gamma$. It is clear that for the small value of the incoherent pumping rate $\Lambda$, the probe field suffers an absorption at the frequency of two-photon resonance. It further shows that as the detuning $\Delta$ increases, the transition from absorption to gain for the probe field requires smaller and smaller $\Lambda$, and this property is corresponding to that in Fig. 2(d). When $\Lambda$ is 


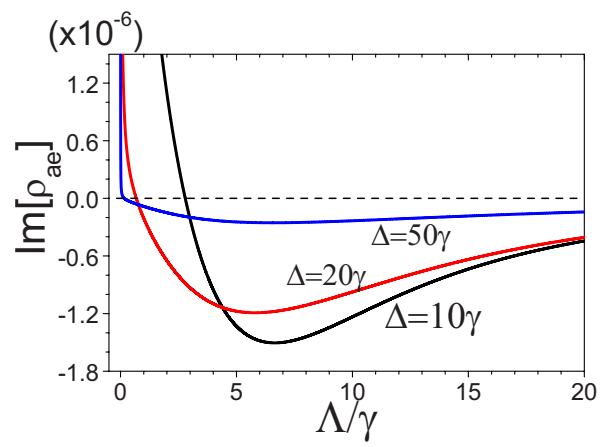

FIG. 3. (Color online) Dependence of the probe gain or absorption $\operatorname{Im}\left[\rho_{a e}\right]$ (at the two-photon resonant frequency $\delta=0$ ) on incoherent pumping rate $\Lambda$ for different detunings: $\Delta=10 \gamma, \Delta=20 \gamma$, and $\Delta=50 \gamma$. Other parameters are the same as in Fig. 2 .

smaller than certain critical value, the gain of the probe field is increasing as $\Lambda$ increases. Above the critical value of $\Lambda$, the gain of the probe field is suppressed as $\Lambda$ increases. Actually, the atom excited from the ground state $|e\rangle$ to the excited state $|a\rangle$ by the incoherent pumping can be moved into the ground states $|b\rangle$ and $|d\rangle$ by the spontaneous decays $\gamma_{d a}$ and $\gamma_{b a}$. This process provides the atomic population transfer. Without the incoherent pumping, we will obtain the corresponding Raman absorption peaks.

Next we discuss how to control the splitting of the Raman gain peaks. On the one hand, the susceptibility $\chi$ can be controlled by changing the intensities of the Rabi frequencies $\Omega_{2}$ and $\Omega_{3}$ (see Figs. 4 and 5). From Figs. 4 and 5, it is seen that for the smaller intensities of $\Omega_{2}$ and $\Omega_{3}$, there is only a single Raman gain peak near the frequency of the twophoton resonance [see Figs. 4(a) and 5(a)]. In this case, the Raman gain peak does not split due to the small values of $\Omega_{2}$ and $\Omega_{3}$. However, for the larger values of $\Omega_{2}$ and $\Omega_{3}$ the Raman gain peak splits into a doublet, which leads to the strong negative dispersion with very less gain near the frequency of the two-photon resonance $\delta=0$. Comparing Fig. 5 with Fig. 4, it is clear that for the larger detuning $\Delta$, the variation of $\chi$ with respect to the two-photon detuning $\delta$ becomes symmetrical due to the diminished effect of the one-photon resonant absorption. It should also be noted that a small shift for the symmetrical center of Raman peaks is

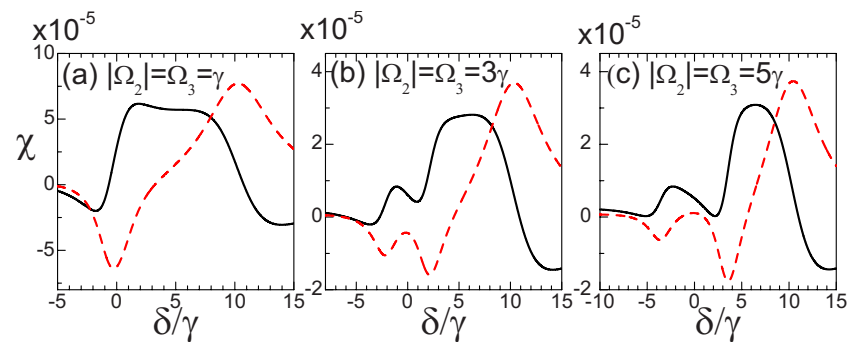

FIG. 4. (Color online) Real (solid line) and imaginary (dashed line) parts of the linear susceptibility $\chi$ vs two-photon detuning $\delta$ for different intensities of the controlling fields (a) $\left|\Omega_{2}\right|=\Omega_{3}=\gamma$, (b) $\left|\Omega_{2}\right|=\Omega_{3}=3 \gamma$, and (c) $\left|\Omega_{2}\right|=\Omega_{3}=5 \gamma$. Other parameters are $\Omega_{1}$ $=\Omega_{4}=2 \gamma, \Lambda=2 \gamma, \Delta=10 \gamma$, and $\phi_{0}=0$.

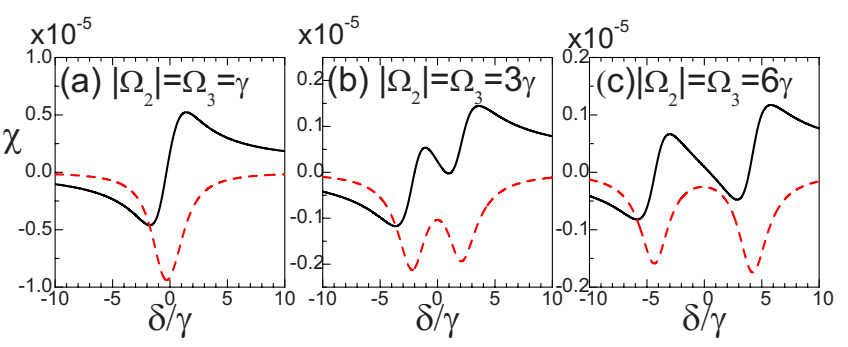

FIG. 5. (Color online) Real (solid line) and imaginary (dashed line) parts of the linear susceptibility $\chi$ vs two-photon detuning $\delta$ for different intensities of the controlling fields (a) $\left|\Omega_{2}\right|=\Omega_{3}=\gamma$, (b) $\left|\Omega_{2}\right|=\Omega_{3}=3 \gamma$, and (c) $\left|\Omega_{2}\right|=\Omega_{3}=6 \gamma$. Other parameters are $\Omega_{1}$ $=\Omega_{4}=5 \gamma, \Lambda=2 \gamma, \Delta=80 \gamma$, and $\phi_{0}=0$.

observed due to the finiteness of the driving fields $\Omega_{1}$ and $\Omega_{4}$ relative to the detuning $\Delta$. Figure 6 shows that there is an optimal intensity control for the largest negative value of $\eta$. It is also clear that as the detuning $\Delta$ becomes larger, the largest negative value of $\eta$ becomes smaller.

The most interesting effect is that the susceptibility $\chi$ could also be changed by adjusting the phase $\phi_{0}$ of the controlling field corresponding to the Rabi frequency $\Omega_{2}$. In Figs. 7 and 8 , we plot the susceptibilities $\chi$ under different phases $\phi_{0}$ for the two cases: $\Delta=10 \gamma$ and $\Delta=80 \gamma$, respectively. It is shown that for the case $\phi_{0}=0$ there are two Raman gain peaks which leads to the negative dispersion (superluminal propagation) with less gain near the two-photon
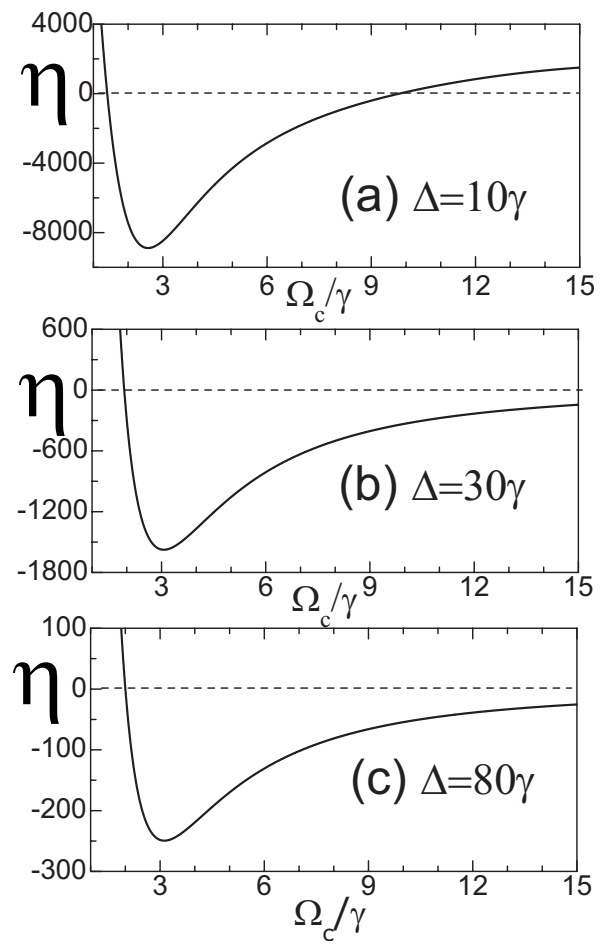

FIG. 6. Variation of the value $\eta$ (related to the group index $n_{g}$ ) with respect to the control field Rabi frequencies $\left|\Omega_{2}\right|=\Omega_{3}=\Omega_{c}$ under different detuning (a) $\Delta=10 \gamma$, (b) $\Delta=30 \gamma$, and (c) $\Delta=80 \gamma$, with $\Omega_{1}=\Omega_{4}=5 \gamma, \Lambda=2 \gamma$. Other parameters are the same as in Fig. 5 . 

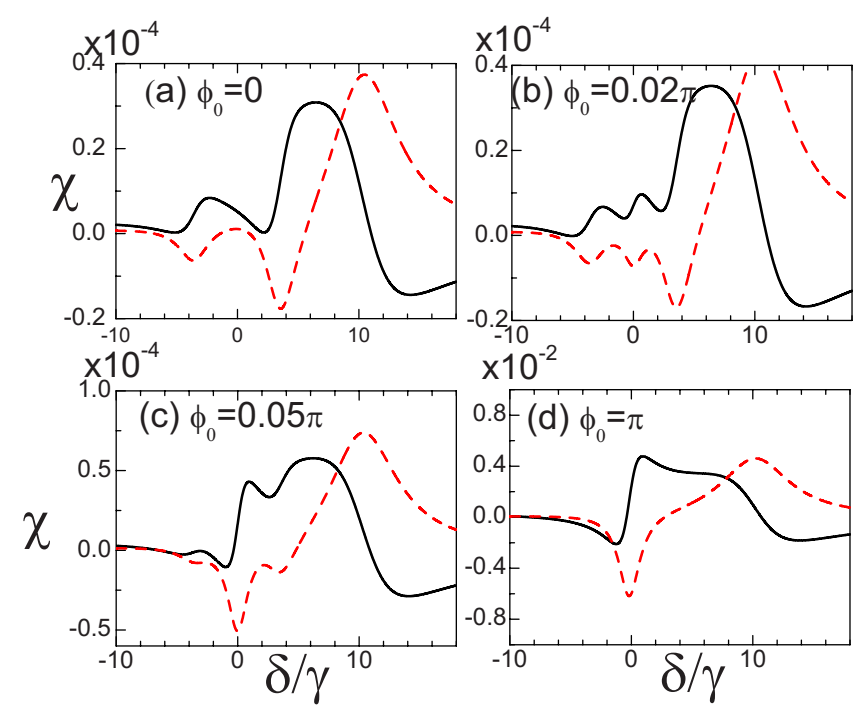

FIG. 7. (Color online) Real (solid line) and imaginary (dashed line) parts of the linear susceptibility $\chi$ vs two-photon detuning $\delta$ for different phases of the controlling fields (a) $\phi_{0}=0$, (b) $\phi_{0}$ $=0.02 \pi$, (c) $\phi_{0}=0.05 \pi$, and (d) $\phi_{0}=\pi$, with $\left|\Omega_{2}\right|=\Omega_{3}=5 \gamma$. Other parameters are the same as in Fig. 4.

resonance $\delta=0$. With the changing of the phase $\phi_{0}$, the susceptibility $\chi$ is changed into three Raman gain peaks [see Figs. 7(b) and 7(c), and Figs. 8(b) and 8(c)]. In this case, the dispersion near the two-photon resonance $\delta=0$ is gradually changed from anomalous to normal dispersion, that is to say, the group velocity of the probe pulse changes from superluminal to subluminal. For the case $\phi_{0}=\pi$, we find that there is only one Raman gain peak. This leads to the extremely slow light propagation in an amplifying medium. In Fig. 9, we show the phase dependence of $\eta$ under different detuning $\Delta$. It is clear that near $\phi_{0}=0$, the value of $\eta$ (related with the group index) could be largely negative, and this indicates
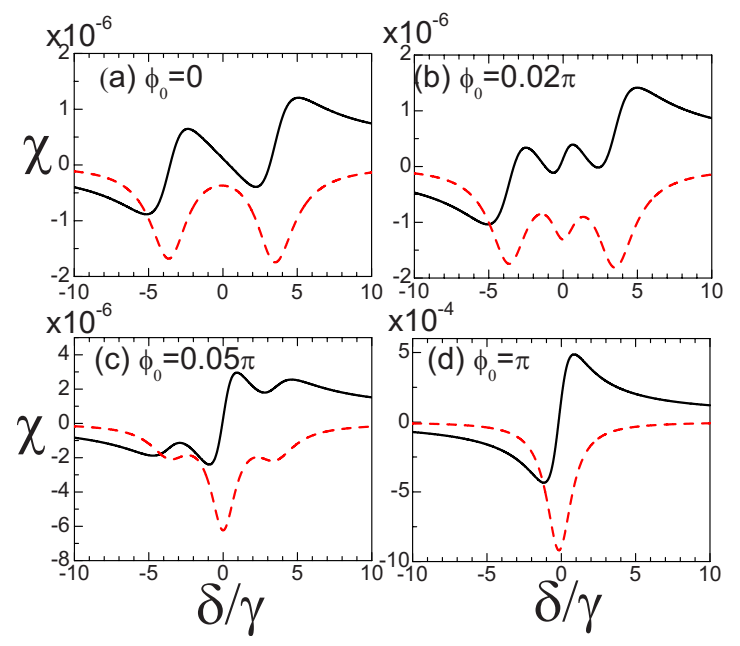

FIG. 8. (Color online) Real (solid line) and imaginary (dashed line) parts of the linear susceptibility $\chi$ vs two-photon detuning $\delta$ for different phases of the controlling fields (a) $\phi_{0}=0$, (b) $\phi_{0}$ $=0.02 \pi$, (c) $\phi_{0}=0.05 \pi$, and (d) $\phi_{0}=\pi$, with $\left|\Omega_{2}\right|=\Omega_{3}=5 \gamma$. Other parameters are the same as in Fig. 5.

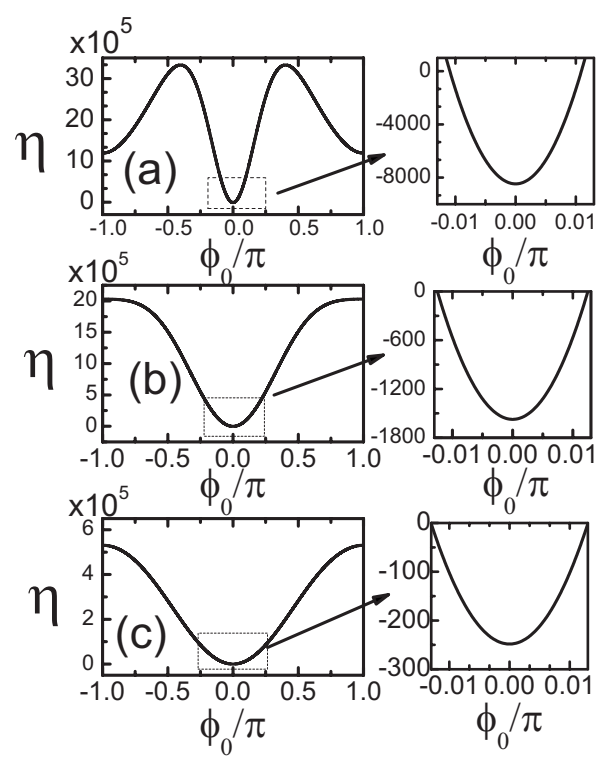

FIG. 9. Dependence of the value $\eta$ (related to the group index $n_{g}$ ) on the phase $\phi_{0}$ of the control field $\Omega_{2}$ under different detuning (a) $\Delta=10 \gamma$, (b) $\Delta=30 \gamma$, and (c) $\Delta=80 \gamma$, with $\Omega_{1}=\Omega_{4}=5 \gamma,\left|\Omega_{2}\right|$ $=\Omega_{3}=3 \gamma$, and $\Lambda=2 \gamma$.

that the propagation of the probe pulse is highly superluminal.

The above result for the phase dependence of the susceptibility can be understood in terms of dressed states, which are defined as the eigenstates of the Hamiltonian $H_{c}$ of the medium associated with the coherent driving fields. We assume $\Omega_{1}=\Omega_{4}=\Omega_{R}$ and $\left|\Omega_{2}\right|=\Omega_{3}=\Omega_{c}$ to be real and $\Delta_{2}=\Delta_{3}$ $=0$. We further assume the detuning $\Delta_{1}=\Delta_{4}=\Delta$ to be sufficiently large [32]. Therefore, we obtain the following effective Hamiltonian for the coherent driving field part:

$$
H_{e f f}=H_{c}+H_{p},
$$

where

$$
\begin{aligned}
H_{c}= & -\frac{\hbar \Omega_{R}^{2}}{4 \Delta}[(|b\rangle\langle b|+| d\rangle\langle d|) / 2-|a\rangle\langle a|]-\frac{\hbar \Omega_{R}^{2}}{4 \Delta}[|b\rangle\langle d|] \\
& -\frac{\hbar}{2} \Omega_{c}\left[|b\rangle\left\langle c\left|e^{i \phi_{0}}+\right| d\right\rangle\langle c|\right]+\text { H.c. }
\end{aligned}
$$

and

$$
\begin{aligned}
H_{p}= & -\frac{\hbar \Omega_{p}^{2}}{4 \Delta}[(|e\rangle\langle e|-| a\rangle\langle a|) / 2]-\frac{\hbar \Omega_{p} \Omega_{R}}{4 \Delta}[|e\rangle\langle b|+| e\rangle\langle d|] \\
& + \text { H.c., }
\end{aligned}
$$

where we have considered $\Delta \approx \Delta_{p}$. Different terms in $H_{c}$ and $H_{p}$ can be identified as ac Stark shifts experienced by the atomic system due to the application of two far off resonance coherent driving fields corresponding to the Rabi frequencies $\Omega_{1}$ and $\Omega_{4}$, the Raman transitions between atomic levels $|b\rangle$ and $|d\rangle$ and resonance transitions corresponding to the Rabi frequencies $\Omega_{2}$ and $\Omega_{3}$. We ignore the terms corresponding to ac Stark shifts that do not play any significant role in the 
analysis of the results and obtain the reduced Hamiltonian $H_{c}$ which can be written in the matrix form as

$$
H_{c}=-\frac{\hbar}{4}\left(\begin{array}{ccc}
0 & \Omega_{c} e^{i \phi_{0}} & \Omega_{R}^{2} / \Delta \\
\Omega_{c} e^{-i \phi_{0}} & 0 & \Omega_{c} \\
\Omega_{R}^{2} / \Delta & \Omega_{c} & 0
\end{array}\right) .
$$

We diagonalize this $3 \times 3$ matrix to obtain dressed states $| \pm\rangle$ and $|0\rangle$ which correspond to the eigenvalues $\lambda_{ \pm, 0}$, respectively. These eigenvalues $\lambda_{ \pm, 0}$ are the three roots of the cubic equation $\lambda^{3}-\left(\frac{\Omega_{R}^{4}}{16 \Delta^{2}}+\frac{\Omega_{c}^{2}}{8}\right) \lambda+\frac{\bar{\Omega}_{R}^{2} \Omega_{c}^{2} \cos \phi_{0}}{32 \Delta}=0$. Therefore, our fivelevel atomic system could be equivalent to the configuration shown in Fig. 1(b). Actually under the condition of the absence of $\Omega_{1}$ and $\Omega_{4}$ or the condition of the large detuning $\Delta$, the splitting of the states $| \pm\rangle$ and $|0\rangle$ is mainly determined by $\Omega_{c} / \sqrt{2}$, similar to Ref. [26]. In the present scheme, because the levels $|b\rangle$ and $|d\rangle$ are connected by the driving fields $\Omega_{1}$ and $\Omega_{4}$ (with the large detuning $\Delta$ ), the different two-photon Raman pathways will produce the quantum interference effect, which leads to the phase-dependent effect of the susceptibility. The dressed states associated with the three eigenvalues can be expressed in terms of the bare states $(|b\rangle,|c\rangle$, and $|d\rangle)$ as follows:

$$
\left(\begin{array}{c}
|+\rangle \\
|0\rangle \\
|-\rangle
\end{array}\right)=\left(\begin{array}{ccc}
\alpha_{+} & \beta_{+} & \eta_{+} \\
\alpha_{0} & \beta_{0} & \eta_{0} \\
\alpha_{-} & \beta_{-} & \eta_{-}
\end{array}\right)\left(\begin{array}{c}
|b\rangle \\
|c\rangle \\
|d\rangle
\end{array}\right),
$$

where the coefficients $\alpha_{ \pm, 0}, \beta_{ \pm, 0}$, and $\eta_{ \pm, 0}$ depend on the values of $\Omega_{R}$ and $\Omega_{c}$, and are especially affected by the phase factor $\phi_{0}$. In the case of $\phi_{0}=0$, these coefficients are given by

$$
\left(\begin{array}{ccc}
\alpha_{+} & \beta_{+} & \eta_{+} \\
\alpha_{0} & \beta_{0} & \eta_{0} \\
\alpha_{-} & \beta_{-} & \eta_{-}
\end{array}\right)=\left(\begin{array}{cccc}
\frac{\Omega_{R}^{2}+\xi}{\sqrt{2\left[\left(\Omega_{R}^{2}+\xi\right)^{2}+8 \Delta^{2} \Omega_{c}^{2}\right]}} & \frac{1}{\sqrt{2}} & \frac{\Omega_{R}^{2}-\xi}{\sqrt{2\left[\left(\Omega_{R}^{2}-\xi\right)^{2}+8 \Delta^{2} \Omega_{c}^{2}\right]}} \\
\frac{2 \sqrt{2} \Delta \Omega_{c}}{\sqrt{\left(\Omega_{R}^{2}+\xi\right)^{2}+8 \Delta^{2} \Omega_{c}^{2}}} & 0 & \frac{2 \sqrt{2} \Delta \Omega_{c}}{\sqrt{2\left[\left(\Omega_{R}^{2}-\xi\right)^{2}+8 \Delta^{2} \Omega_{c}^{2}\right]}} \\
\frac{\Omega_{R}^{2}+\xi}{\sqrt{2\left[\left(\Omega_{R}^{2}+\xi\right)^{2}+8 \Delta^{2} \Omega_{c}^{2}\right]}} & -\frac{1}{\sqrt{2}} & \frac{\Omega_{R}^{2}-\xi}{\sqrt{2\left[\left(\Omega_{R}^{2}-\xi\right)^{2}+8 \Delta^{2} \Omega_{c}^{2}\right]}}
\end{array}\right),
$$

where $\xi=\sqrt{\Omega_{R}^{4}+8 \Delta^{2} \Omega_{c}^{2}}$. For the case of $\phi_{0}=\pi$, these coefficients change into

$$
\left(\begin{array}{ccc}
\alpha_{+} & \beta_{+} & \eta_{+} \\
\alpha_{0} & \beta_{0} & \eta_{0} \\
\alpha_{-} & \beta_{-} & \eta_{-}
\end{array}\right)=\left(\begin{array}{cccc}
\frac{\Omega_{R}^{2}-\xi}{\sqrt{2\left[\left(\Omega_{R}^{2}-\xi\right)^{2}+8 \Delta^{2} \Omega_{c}^{2}\right]}} & \frac{1}{\sqrt{2}} & \frac{\Omega_{R}^{2}+\xi}{\sqrt{2\left[\left(\Omega_{R}^{2}+\xi\right)^{2}+8 \Delta^{2} \Omega_{c}^{2}\right]}} \\
\frac{2 \sqrt{2} \Delta \Omega_{c}}{\sqrt{\left(\Omega_{R}^{2}-\xi\right)^{2}+8 \Delta^{2} \Omega_{c}^{2}}} & 0 & \frac{2 \sqrt{2} \Delta \Omega_{c}}{\sqrt{2\left[\left(\Omega_{R}^{2}+\xi\right)^{2}+8 \Delta^{2} \Omega_{c}^{2}\right]}} \\
-\frac{\Omega_{R}^{2}-\xi}{\sqrt{2\left[\left(\Omega_{R}^{2}-\xi\right)^{2}+8 \Delta^{2} \Omega_{c}^{2}\right]}} & \frac{1}{\sqrt{2}} & -\frac{\Omega_{R}^{2}+\xi}{\sqrt{2\left[\left(\Omega_{R}^{2}+\xi\right)^{2}+8 \Delta^{2} \Omega_{c}^{2}\right]}}
\end{array}\right) .
$$

Under the same assumptions as are considered for coherent part of the effective Hamiltonian $H_{c}$, the coupling Hamiltonian $H_{p}$ of the medium associated with the probe field in an appropriate frame is simply given by

$$
H_{p}=-\frac{\hbar \Omega_{p} \Omega_{R}}{4 \Delta}[|e\rangle\langle b|+| e\rangle\langle d|+| b\rangle\langle e|+| d\rangle\langle e|] .
$$

Writing the bare states in terms of dressed states

$$
\left(\begin{array}{l}
|b\rangle \\
|c\rangle \\
|d\rangle
\end{array}\right)=\left(\begin{array}{ccc}
\alpha_{+} & \beta_{+} & \eta_{+} \\
\alpha_{0} & \beta_{0} & \eta_{0} \\
\alpha_{-} & \beta_{-} & \eta_{-}
\end{array}\right)\left(\begin{array}{c}
|+\rangle \\
|0\rangle \\
|-\rangle
\end{array}\right),
$$

from Eq. (19) we can rewrite $H_{p}$ for the case $\phi_{0}=0$ as

$$
H_{p}=-\frac{\hbar \Omega_{p} \Omega_{R}}{2 \Delta}\left(\mu_{1}|e\rangle\left\langle+\left|+\mu_{2}\right| e\right\rangle\left\langle-\left|+\mu_{1}\right|+\right\rangle\left\langle e\left|+\mu_{2}\right|-\right\rangle\langle e|\right),
$$

where

$$
\mu_{1}=\frac{\Omega_{R}^{2}+\xi}{\sqrt{2\left[\left(\Omega_{R}^{2}-\xi\right)^{2}+8 \Delta^{2} \Omega_{c}^{2}\right]}}
$$

and

$$
\mu_{2}=\frac{\Omega_{R}^{2}-\xi}{\sqrt{2\left[\left(\Omega_{R}^{2}-\xi\right)^{2}+8 \Delta^{2} \Omega_{c}^{2}\right]}} .
$$

In Eq. (24), we find that the transition from the dressed state $|0\rangle$ to $|e\rangle$ disappears due to the fact that the state $|0\rangle$ becomes a dark state $|0\rangle=(|b\rangle-|d\rangle) / \sqrt{2}$, which indicates the destruc- 
tive quantum interference between two Raman pathways from $|b\rangle$ and $|d\rangle$ to $|e\rangle$. Whereas the other two dressed states are given by

$$
\begin{aligned}
| \pm\rangle= & \frac{\Omega_{R}^{2} \pm \xi}{\sqrt{2\left[\left(\Omega_{R}^{2}-\xi\right)^{2}+8 \Delta^{2} \Omega_{c}^{2}\right]}}|b\rangle \\
& +\frac{\Omega_{R}^{2} \pm \xi}{\sqrt{2\left[\left(\Omega_{R}^{2}-\xi\right)^{2}+8 \Delta^{2} \Omega_{c}^{2}\right]}}|d\rangle \\
& +\frac{2 \sqrt{2} \Delta \Omega_{c}}{\sqrt{\left(\Omega_{R}^{2} \pm \xi\right)^{2}+8 \Delta^{2} \Omega_{c}^{2}}}|c\rangle,
\end{aligned}
$$

which lead to the enhanced Raman gain peaks due to the constructive quantum interference between two Raman pathways from $|b\rangle$ and $|d\rangle$ to $|e\rangle$. Therefore, there are only two transitions from the dressed states $| \pm\rangle$ to the state $|e\rangle$ in the dressed-state picture, which correspond to two sideward Raman gain peaks. It should be emphasized that the mechanism of the existence for two sideward Raman gain peaks here is very different from the proposal by Agarwal et al. [17] where the two Raman gain peaks are controlled by the intensity of the coherent controlling field without the process of the quantum interference between different two-photon pathways.

In the case of $\phi_{0}=\pi$, the coupling Hamiltonian $H_{p}$ can be rewritten as

$$
H_{p}=-\frac{\hbar \Omega_{p} \Omega_{R}}{4 \Delta}(\sqrt{2}|e\rangle\langle 0|+\sqrt{2}| 0\rangle\langle e|) .
$$

In this case, we find that two transitions from $| \pm\rangle$ to $|e\rangle$ disappear because these two dressed states become the coherent superposition states

$$
\begin{aligned}
| \pm\rangle= & \frac{\Omega_{R}^{2} \mp \xi}{\sqrt{2\left[\left(\Omega_{R}^{2} \mp \xi\right)^{2}+8 \Delta^{2} \Omega_{c}^{2}\right]}}|b\rangle \\
& -\frac{\Omega_{R}^{2} \mp \xi}{\sqrt{2\left[\left(\Omega_{R}^{2} \mp \xi\right)^{2}+8 \Delta^{2} \Omega_{c}^{2}\right]}}|d\rangle \\
& +\frac{2 \sqrt{2} \Delta \Omega_{c}}{\sqrt{\left(\Omega_{R}^{2} \mp \xi\right)^{2}+8 \Delta^{2} \Omega_{c}^{2}}}|c\rangle,
\end{aligned}
$$

which lead to the canceling effect between the two Raman pathways from $|b\rangle$ and $|d\rangle$ to $|e\rangle$; while for the dressed state $|0\rangle$, it becomes $|0\rangle=(|b\rangle+|d\rangle) / \sqrt{2}$, which leads to the enhanced Raman gain peak due to the constructive quantum interference. Therefore, there is only one transition from the state $|0\rangle$ to $|e\rangle$ corresponding to single Raman gain peak in Fig. 7(d) and Fig. 8(d).

In a general case, i.e., for any value of $\phi_{0}$ between 0 and $\pi$, the Hamiltonian $H_{p}$ from Eq. (22) can always be rewritten as

$$
\begin{aligned}
H_{p}= & -\frac{\hbar \Omega_{p} \Omega_{R}}{4 \Delta}\left[\left(\alpha_{+}+\alpha_{-}\right)|e\rangle\left\langle+\left|+\left(\beta_{+}+\beta_{-}\right)\right| e\right\rangle\langle 0|+\left(\eta_{+}+\eta_{-}\right)\right. \\
& \times|e\rangle\langle-|+\text { H.c. }],
\end{aligned}
$$

where $\left(\alpha_{+}+\alpha_{-}\right),\left(\beta_{+}+\beta_{-}\right)$, and $\left(\eta_{+}+\eta_{-}\right)$do not equal to zero. Therefore, there exist three Raman gain peaks in the curves of the susceptibility [see Figs. 7(b), 7(c), 8(b), and 8(c)].

In conclusion, we have presented a Raman scheme in a five-level atomic system. We find that the Raman process is greatly affected by the incoherent pump, which leads to the propagation of the probe pulse from the subluminal to superluminal with less absorption or gain. We further demonstrate the possibility of the Raman process controlled by adjusting the intensities and phases of the controlling fields, and show the dependence of the susceptibility and the group index (indicated by the value of $\eta$ ) on the intensities and the collective phases of the controlling fields. Meanwhile, we also have qualitatively explained the phase dependence of the Raman gain processes on the basis of the dressed states.

L.G.W. would like to acknowledge the hospitality at COMSATS Institute of Information Technology where this work was done and the support from NSFC (Contract No. 10604047). We would also like to acknowledge COMSTECH for its support.
[1] R. Y. Chiao and A. M. Steinberg, in Progress in Optics, edited by E. Wolf (Elsevier, Amsterdam, 1997), Vol. 37, p. 345; R. W. Boyd and D. J. Gauthier, in Progress in Optics, edited by E. Wolf (Elsevier, Amsterdam, 2002), Vol. 43, p. 497.

[2] P. W. Milloni, J. Phys. B 35, R31 (2002).

[3] K. Kim, H. S. Moon, C. Lee, S. K. Kim, and J. B. Kim, Phys. Rev. A 68, 013810 (2003).

[4] S. E. Harris, J. E. Field, and A. Kasapi, Phys. Rev. A 46, R29 (1992).

[5] L. V. Hau, S. E. Harris, Z. Dutton, and C. H. Behroozi, Nature (London) 397, 594 (1999).

[6] A. Kasapi, M. Jain, G. Y. Yin, and S. E. Harris, Phys. Rev. Lett. 74, 2447 (1995).
[7] M. Xiao, Y.-Q. Li, S.-Z. Jin, and J. Gea-Banacloche, Phys. Rev. Lett. 74, 666 (1995).

[8] M. M. Kash, V. A. Sautenkov, A. S. Zibrov, L. Hollberg, G. R. Welch, M. D. Lukin, Y. Rostovtsev, E. S. Fry, and M. O. Scully, Phys. Rev. Lett. 82, 5229 (1999).

[9] D. Budker, D. F. Kimball, S. M. Rochester, and V. V. Yashchuk, Phys. Rev. Lett. 83, 1767 (1999).

[10] A. V. Turukhin, V. S. Sudarshanam, M. S. Shahriar, J. A. Musser, B. S. Ham, and P. R. Hemmer, Phys. Rev. Lett. 88, 023602 (2001).

[11] M. S. Bigelow, N. N. Lepeshkin, and R. W. Boyd, Phys. Rev. Lett. 90, 113903 (2003).

[12] A. M. Steinberg and R. Y. Chiao, Phys. Rev. A 49, 2071 
(1994).

[13] L. J. Wang, A. Kuzmich, and A. Dogariu, Nature (London) 406, 277 (2000); A. Dogariu, A. Kuzmich, and L. J. Wang, Phys. Rev. A 63, 053806 (2001).

[14] Md. Aminul Islam Talukder, Y. Amagishi, and M. Tomita, Phys. Rev. Lett. 86, 3546 (2001).

[15] Y. Shimizu, N. Shiokawa, N. Yamamoto, M. Kozuma, T. Kuga, L. Deng, and E. W. Hagley, Phys. Rev. Lett. 89, 233001 (2002)

[16] G. S. Agarwal, T. N. Dey, and S. Menon, Phys. Rev. A 64, 053809 (2001).

[17] G. S. Agarwal and S. Dasgupta, Phys. Rev. A 70, 023802 (2004).

[18] V. S. C. Manga Rao, S. Dutta Gupta, and G. S. Agarwal, Opt. Lett. 29, 307 (2004).

[19] L.-G. Wang, H. Chen, and S.-Y. Zhu, Phys. Rev. E 70, 066602 (2004).

[20] D. Bortman-Arbiv, A. D. Wilson-Gordon, and H. Friedmann, Phys. Rev. A 63, 043818 (2001).

[21] M. Sahrai, H. Tajalli, K. T. Kapale, and M. Suhail Zubairy, Phys. Rev. A 70, 023813 (2004).

[22] M. Mahmoudi, M. Sahrai, and H. Tajalli, J. Phys. B 39, 1825
(2006); Phys. Lett. A, 357, 66 (2006).

[23] K. J. Jiang, L. Deng, and M. G. Payne, Phys. Rev. A 74, 041803(R) (2006).

[24] L. Li, H. Guo, F. Xiao, X. Peng, and X. Chen, J. Opt. Soc. Am. B 22, 1309 (2005).

[25] X.-M. Hu, D. Du, G.-L. Cheng, J.-H. Zou, and X. Li, J. Phys. B 38, 827 (2005).

[26] X.-M. Hu and J. Xu, Phys. Rev. A 69, 043812 (2004).

[27] M. Mahmoudi and J. Evers, Phys. Rev. A 74, 063827 (2006).

[28] M. O. Scully and M. S. Zubairy, Quantum Optics (Cambridge University Press, Cambridge, U.K., 1997).

[29] X.-M. Hu, G.-L. Cheng, J.-H. Zou, X. Li, and D. Du, Phys. Rev. A 72, 023803 (2005).

[30] M. Fleischhauer, C. H. Keitel, M. O. Scully, C. Su, B. T. Ulrich, and S.-Y. Zhu, Phys. Rev. A 46, 1468 (1992); M. Fleischhauer, C. H. Keital, M. O. Scully, and C. Su, Opt. Commun. 87, 109 (1992).

[31] S. Sultana and M. S. Zubairy, Phys. Rev. A 49, 438 (1994).

[32] H. Xiong, M. O. Scully, and M. S. Zubairy, Phys. Rev. Lett. 94, 023601 (2005); G.-X. Li and J.-S. Peng, Phys. Rev. A 52, 465 (1995). 